\title{
A Conventional Proof of Kerr's Theorem*
}

\author{
D. Cox and E. J. Flaherty, Jr. $\star \star$ \\ Center for Relativity Theory, The University of Texas at Austin, Austin, Texas, USA
}

\begin{abstract}
A proof of Kerr's Theorem for generating geodesic and shearfree null congruences in Minkowski space is given in the Newman-Penrose null tetrad formalism.
\end{abstract}

Kerr's Theorem gives an algorithm for obtaining the most general geodesic and shearfree null congruence (GSF congruence) in Minkowski space. The theorem is important for constructing the Kerr-Schild spacetimes [1] and is also essential in the twistor treatment of zero rest mass free fields in Minkowski space [2]. The published proofs of Kerr's Theorem must be either extracted indirectly from the Kerr-Schild field equations [1], or they must be translated from twistor language which is unfamiliar to many relativists [2]. Nowhere, to our knowledge, is there to be found a straightforward proof of the theorem in familiar terms. The purpose of this note is to remedy this situation by presenting a proof of Kerr's Theorem in the Newman-Penrose null tetrad formalism [3].

Let the metric of Minkowski space be written as

$$
d s^{2}=d u d v-d \zeta d \bar{\zeta}=\left(l_{a} n_{b}+n_{a} l_{b}-m_{a} \bar{m}_{b}-\bar{m}_{a} m_{b}\right) d x^{a} d x^{b},
$$

where $l_{a}, n_{a}, m_{a}, \bar{m}_{a}$ is a constant normed null tetrad. Then Kerr's Theorem states that the most general analytic GSF null congruence $\xi_{a}$ is given by either $\xi_{a}=$ $n_{a}$ or by

$$
\xi_{a}=l_{a}+Y \bar{m}_{a}+\bar{Y} m_{a}+Y \bar{Y} n_{a},
$$

where $\bar{Y}$ is a complex function of the coordinates $u, v, \zeta, \bar{\zeta}$ defined implicitly by $F=0$, where

$$
F=F(\bar{Y}, u+\bar{Y} \zeta, \bar{\zeta}+\bar{Y} v)
$$

is an arbitrary complex analytic function of its three arguments. [The case $\xi_{a}=n_{a}$ can be thought of as arising from the form (2) in the limit $Y \rightarrow \infty$.]

* Supported in part by NSF Grant GP-43844-X.

$\star \star$ Present address: Department of Physics, Syracuse University, 201 Physics Building, Syracuse, New York 13210, USA. 
The first step to recall that the GSF property is a function of the directions only of the vectors of the null congruence, i.e. $\alpha \xi_{a}$ is GSF if $\xi_{a}$ is GSF. Then it follows that (2) sweeps out every null direction except $n_{a}$ as $Y$ is varied. This is because the most general null direction is given by

$$
\xi_{a}=a l_{a}+b \bar{m}_{a}+c m_{a}+d n_{a}
$$

with some functions $a, b, c, d$ with

$$
\xi_{a} \xi^{a}=0=a d-b c \quad \text { with } \quad a=\bar{a}, \quad d=\bar{d}, \quad b=\bar{c}
$$

for a real null direction. If $a \neq 0$, we can choose the scaling such that

$$
\xi_{a}=l_{a}+b^{\prime} \bar{m}_{a}+c^{\prime} m_{a}+d^{\prime} n_{a} .
$$

Then (4) gives

$$
\begin{aligned}
d^{\prime}-b^{\prime} c^{\prime} & =0 \\
d^{\prime} & =b^{\prime} c^{\prime}=c^{\prime} \vec{c} .
\end{aligned}
$$

Then setting $c^{\prime}=\bar{Y}$ gives the desired form (2). If $a=0$, then $b c=0$ by (4) and the case $\xi_{a}=n_{a}$ is obtained.

We can now look upon $\xi_{a}$ in the form (2) as the new tetrad leg $\hat{l}_{a}$ resulting from a "null rotation" about $n_{a}[4]$ :

$$
\begin{aligned}
l_{a} \rightarrow \hat{l}_{a} & =l_{a}+Y \bar{m}_{a}+\bar{Y} m_{a}+Y \bar{Y} n_{a} \\
m_{a} \rightarrow \hat{m}_{a} & =m_{a}+Y n_{a} \\
\bar{m}_{a} \rightarrow \hat{m}_{a} & =\bar{m}_{a}+\bar{Y} n_{a} \\
n_{a} \rightarrow \hat{n}_{a} & =n_{a} .
\end{aligned}
$$

Under such a null rotation, Kinnersley [6] has shown that the spin coefficients $x$ and $\sigma$ transform according to

$$
\begin{aligned}
x \rightarrow \hat{\chi}= & x+\bar{Y} \sigma+Y(\varrho+2 \varepsilon) \\
& +Y \bar{Y}(2 \beta+\tau)+Y^{2}(\pi+2 \alpha) \\
& +Y^{2} \bar{Y}(\mu+2 \gamma)+Y^{3} \lambda \\
& +Y^{3} \bar{Y} v-D Y-\bar{Y} \delta Y \\
& -Y \bar{\delta} Y-Y \bar{Y} \Delta Y, \\
\sigma \rightarrow \hat{\sigma}= & \sigma+Y(\tau+2 \beta)+Y^{2}(\mu+2 \gamma)+Y^{3} v-\delta Y-Y \Delta Y .
\end{aligned}
$$

Under our assumption of a constant null tetrad, with respect to the null coordinates of (1) (which can occur only for Minkowski space), all the spin coefficients must vanish. Then (8) becomes

$$
\begin{aligned}
& x \rightarrow \hat{\varkappa}=-D Y-\bar{Y} \delta Y-Y \bar{\delta} Y-Y \bar{Y} \Delta Y \\
& \sigma \rightarrow \hat{\sigma}=-\delta Y-Y \Delta Y .
\end{aligned}
$$


There is no loss of generality in choosing the constant tetrad in such a way that in the coordinate system $u, v, \zeta, \bar{\zeta}$, we have:

$$
\begin{gathered}
l_{a}=(1,0,0,0) \\
n_{a}=(0,1,0,0) \\
m_{a}=(0,0,1,0) \\
\bar{m}_{a}=(0,0,0,1) .
\end{gathered}
$$

So that

$$
\begin{aligned}
& D=\partial_{v} \\
& \Delta=\partial_{u} \\
& \delta=-\partial_{\bar{\zeta}} \\
& \bar{\delta}=-\partial_{\zeta} .
\end{aligned}
$$

Then (8) becomes

$$
\begin{aligned}
\hat{x} & =-\partial_{v} Y+\bar{Y} \partial_{\bar{\zeta}} Y+Y \partial_{\zeta} Y-Y \bar{Y} \partial_{u} Y \\
& =-\left(\partial_{v}-Y \partial_{\zeta}\right) Y+\bar{Y}\left(\partial_{\bar{\zeta}}-Y \partial_{u}\right) Y, \\
\hat{\sigma} & =+\partial_{\bar{\zeta}} Y-Y \partial_{u} Y \\
& =\left(\partial_{\bar{\zeta}}-Y \partial_{u}\right) Y .
\end{aligned}
$$

To apply the GSF conditions, we set $\hat{x}=\hat{\sigma}=0$. The differential equations which result are equivalent to the following, obtained from (11) with $\hat{x}=\hat{\sigma}=0$ by taking appropriate complex conjugates and linear combinations:

$$
\begin{aligned}
& \left(\partial_{v}-\bar{Y} \partial_{\bar{\zeta}}\right) \bar{Y}=0 \\
& \left(\partial_{\zeta}-\bar{Y} \partial_{u}\right) \bar{Y}=0 .
\end{aligned}
$$

Now we consider the system of equations

$$
\begin{aligned}
& \left(\partial_{v}-\bar{Y} \partial_{\bar{\zeta}}\right) X=0 \\
& \left(\partial_{\zeta}-\bar{Y} \partial_{u}\right) X=0
\end{aligned}
$$

where for the moment $Y$ is considered to be known. This is a system of linear partial differential equations. A necessary and sufficient condition for this system to be completely integrable, i.e. for two independent solutions to exist, is given by Eisenhart [5, pp. 69-70]. This integrability condition is simply that the commutator of the two linear differential operators $\left(\partial_{v}-\bar{Y} \partial_{\bar{\zeta}}\right)$ and $\left(\partial_{\zeta}-\bar{Y} \partial_{u}\right)$ should take the form

$$
\left[\left(\partial_{\zeta}-\bar{Y} \partial_{u}\right),\left(\partial_{v}-\bar{Y} \partial_{\bar{\zeta}}\right)\right] \phi=a\left(\partial_{\zeta}-\bar{Y} \partial_{u}\right) \phi+b\left(\partial_{v}-\bar{Y} \partial_{\bar{\zeta}}\right) \phi
$$

for any function $\phi$, where $a, b$, are some functions.

Working out this commutator, we find:

$$
\begin{aligned}
& {\left[\left(\partial_{\zeta}-\bar{Y} \partial_{u}\right),\left(\partial_{v}-\bar{Y} \partial_{\bar{\zeta}}\right)\right] \phi} \\
& \quad=\left(\bar{Y} \bar{Y},{ }_{u} \partial_{\bar{\zeta}}-\bar{Y},{ }_{\zeta} \partial_{\bar{\zeta}}-\bar{Y} \bar{Y},{ }_{\bar{\zeta}} \partial_{u}+\bar{Y},{ }_{v} \partial_{u}\right) \phi \\
& \quad=\left(\partial_{u} \phi\right)\left(\partial_{v}-\bar{Y} \partial_{\bar{\zeta}}\right) \bar{Y}-\left(\partial_{\bar{\zeta}} \phi\right)\left(\partial_{\zeta}-\bar{Y} \partial_{u}\right) \bar{Y} .
\end{aligned}
$$


The only way for this expression to be of the form (14) for arbitrary $\phi$ is to have

$$
\begin{aligned}
& \left(\partial_{v}-\bar{Y} \partial_{\bar{\zeta}}\right) \bar{Y}=0 \\
& \left(\partial_{\zeta}-\bar{Y} \partial_{u}\right) \bar{Y}=0,
\end{aligned}
$$

that is to say, $\bar{Y}$ must satisfy (12).

So now we assume that $\bar{Y}$ is indeed a solution of (12). (Clearly at least one such solution, $Y=$ constant, exists.) Then the system (13) is integrable. Two independent solutions are easily seen to be

$$
\begin{aligned}
& X_{1}=u+\bar{Y} \zeta \\
& X_{2}=\bar{\zeta}+\bar{Y}_{v} .
\end{aligned}
$$

Then the most general (analytic) solution must be given by

$$
X=f\left(X_{1}, X_{2}\right)=f(u+\bar{Y} \zeta, \bar{\zeta}+\bar{Y} v),
$$

where $f$ is an arbitrary analytic function of its arguments.

In particular, the solution $\bar{Y}$ must be expressible in the form (18):

$$
\bar{Y}=g(u+\bar{Y} \zeta, \bar{\zeta}+\bar{Y} v) \Leftrightarrow \bar{Y}-g(u+\bar{Y} \zeta, \bar{\zeta}+\bar{Y} v)=0 \text {. }
$$

Clearly, no generality is lost (and none is gained) by allowing $\bar{Y}$ to be defined implicitly by any analytic function

$$
F(\bar{Y}, u+\bar{Y} \zeta, \bar{\zeta}+\bar{Y} v)=0 .
$$

Thus Kerr's Theorem is proven.

To see the connection with twistor theory, consider a null twistor

$$
\begin{aligned}
Z_{\alpha} & =\left(Z_{0}, Z_{1}, Z_{2}, Z_{3}\right) \\
& =\left(O_{0}, O_{1},-i X^{A O^{\prime}} O_{A^{\prime}},-i X^{A 1^{\prime}} O_{A}\right) \\
& =\left(O_{A^{\prime}},-i X^{B A^{\prime}} O_{B}\right) .
\end{aligned}
$$

We go to a spin frame where

$$
O_{A}=(1, \bar{Y})
$$

and

$$
X^{A A^{\prime}}=\left(\begin{array}{ll}
u & \zeta \\
\bar{\zeta} & v
\end{array}\right),
$$

so that

$$
\begin{aligned}
X^{B A^{\prime}} O_{B} & =\left(\begin{array}{ll}
u & \zeta \\
\bar{\zeta} & v
\end{array}\right)\left(\begin{array}{l}
1 \\
\bar{Y}
\end{array}\right) \\
& =\left(\begin{array}{l}
u+\bar{Y} \zeta \\
\bar{\zeta}+Y v
\end{array}\right) .
\end{aligned}
$$

Then we have

$$
Z_{\alpha}=(1, \bar{Y},-i(u+\bar{Y} \zeta),-i(\bar{\zeta}+\bar{Y} v)),
$$


and if we set

$$
f\left(Z_{\alpha}\right)=\left(Z_{0}\right)^{-n-2} F\left(Z_{1} / Z_{0}, i Z_{2} / Z_{0}, i Z_{3} / Z_{0}\right),
$$

then $f\left(Z_{\alpha}\right)$ is homogeneous of degree $-n-2$ in $Z_{\alpha^{\prime}}$ and $l_{a}=O_{A} \bar{O}_{A^{\prime}}$ is GSF if and only if $f\left(Z_{\alpha}\right)=0$ for some $f$. The corresponding function $F$ then defines a complex analytic surface $F=0$ in the projective twistor space $C$ [2].

Note Added in Proof. Since this paper was written, still another proof of Kerr's Theorem has appeared in a preprint titled "A Complex Minkowski Space Approach to Twistors", by R. O. Hansen and E. T. Newman. Their proof, although entirely different from ours, is also understandable without reference to twistor theory.

\section{References}

1. Debney, G. C., Kerr, R. P., Schild, A.: J. Math. Phys. 10, 1842 (1969)

2. Penrose, R.: Int. J. Theor. Phys. 1, 61 (1968)

3. Newman, E.T., Penrose, R.: J. Math. Phys. 3, 566 (1962)

4. Janis, A.I., Newman, E. T.: J. Math. Phys. 6, 902 (1965)

5. Eisenhart,L.P.: Riemannian Geometry. Princeton University Press 1966

6. Kinnersley, W. M.: Ph.D. Dissertation, California Institute of Technology, unpublished

Communicated by J. Ehlers

(Received September 23, 1974) 
